\title{
Study on Inflammation and Rehabilitation of Patients with Coronary Heart Disease after Percutaneous Coronary Intervention
}

\author{
HUI NI, T. WANG ${ }^{1}$, L. YANG, M. LOU, YAN ZHAO AND LILI WANG ${ }^{*}$ \\ Department of Cardiology, Xuzhou Central Hospital, Xuzhou City 221009, ${ }^{1}$ Department of Emergency, Xuzhou Central \\ Hospital, Xuzhou City 221009, ²Department of Nursing management, Xuzhou Central Hospital, Xuzhou City 221009, China
}

Ni et al.: Rehabilitation of patients with coronary heart disease after percutaneous coronary intervention

\begin{abstract}
In order to prevent the inflammatory reaction caused by vascular wall injury after percutaneous coronary intervention treatment, affecting the therapeutic effect and rehabilitation, this study will select 54 patients with coronary heart disease and then randomly divided them into two groups to observe and compare inflammatory factors and their connection with postoperative thrombolysis in myocardial infarction frame count, the factors include interleukin-6, interleukin-9, interleukin-17A. It has been found that part of factors was elevated after surgery of elective percutaneous coronary intervention for stable coronary heart disease patients, suggesting that percutaneous coronary intervention may induce inflammatory reactions. High level of local inflammatory factors interleukin-6, helper $T$ cells 17 may affect the forward arterial blood flow after interventional intervention. Thrombus aspiration can reduce the occurrence of slow or no reflow in patients with thrombotic load, increase coronary perfusion, improve postoperative cardiac function, and is expected to reduce heart attacks. In patients with coronary heart disease, the expression of inflammatory factors increases with the severity of coronary heart disease and the severity of coronary atherosclerosis. The level of inflammatory factors is correlated with the degree of coronary artery disease, which may promote the progression of coronary lesions. Rehabilitation therapy of traditional Chinese and Western medicine is necessary for patients with coronary heart disease after percutaneous coronary intervention. Active rehabilitation therapy is of great significance in improving the patient's exercise tolerance, reducing the incidence of cardiovascular adverse events, reducing complications and improving patient's quality of life.
\end{abstract}

Key words: Coronary heart disease, percutaneous coronary intervention, inflammatory factors, rehabilitation therapy

The incidence of coronary heart disease (CHD) has increased year by year, and it has become a common disease and frequently-occurring disease ${ }^{[1]}$. More men than women are affected, accounting for $10 \%$ to $20 \%$ of heart disease deaths in China. With the development of cardiovascular interventional medicine, percutaneous coronary intervention (PCI) has become an important means of treatment of coronary artery disease ${ }^{[2]}$, and the rehabilitation of CHD has also progressed from the rehabilitation of traditional myocardial infarction to the rehabilitation of interventional therapy of CHD. Rehabilitation treatment is of great significance for improving the quality of life of patients with CHD after PCI from physiological, psychological and social perspectives. Studies have shown that patients after PCI are suitable for cardiac rehabilitation ${ }^{[3,4]}$. Rehabilitation therapy for CHD after intervention has been paid more and more attention, and its efficacy has been confirmed by many studies. After cardiac rehabilitation, patients with PCI can significantly increase exercise capacity after treatment, significantly improve cardiac function, reduce myocardial oxygen consumption, reduce blood lipid levels, significantly reduce restenosis, and reduce complications. However, complications such as coronary restenosis and myocardial ischemia may occur after interventional therapy, as well as problems such as decreased quality of life and psychological disorders. How to avoid these problems, so that patients with PCI can get better recovery and reduce the occurrence of acute events is the urgent problem

*Address for correspondence

E-mail: wangl|93870036@163.com 
that we need to solve ${ }^{[5]}$. As a minimally invasive method for the treatment of CHD, PCI has achieved rapid development in the past $30 \mathrm{y}$ and has become one of the main methods for the treatment of CDH. PCI has experienced 4 major technological revolutions in the process of development ${ }^{[6]}$. The four technological revolutions of PCI are undoubtedly great and farreaching, but each change is limited to the material and coating of the stent, and there is no change in the shape of the stent, which is always a traditional cylindrical stent. It is well known that the shape of coronary vessels is not cylindrical, but like a branch, the lumen is tapered from near to $\operatorname{far}^{[7]}$. Therefore, placement of a cylindrical stent in a conical coronary artery will inevitably lead to incomplete expansion of the proximal vascular lumen or excessive expansion of the distal vascular lumen, increasing the risk of stent thrombosis and instent restenosis after PCI, and ultimately increasing the major adverse cardiovascular events. Therefore, this study will change the previous use of conical stents to observe the effects of inflammation and rehabilitation after therapy ${ }^{[8,9]}$. In summary, this study aims to inhibit inflammation and reduce other complications and achieve better rehabilitation effect after PCI in patients with $\mathrm{CDH}$ through a specific conical stent. 54 patients (aged 35-80 y) with CHD in Xuzhou Central Hospital from December 2015 to December 2018 are selected in this study and then randomly divided them into two groups: group A and group B (27 patients in each group). The first group (group A) was for patients who underwent elective PCI within 7-14 d of acute coronary syndromes (ACS). The second group (group B) was for patients who underwent routine coronary angiography. The informed consent signed by the patients or their family members was obtained and this study was approved by the medical ethics committee of Xuzhou Central Hospital. Exclusion criteria: All patients except direct PCI within 1 mo or within $24 \mathrm{~h}$ of acute myocardial infarction; highrisk patients in the risk stratification of CHD patients; coronary revascularization due to restenosis; patients concurrent with heart failure, Cardiogenic shock, severe cardiopulmonary insufficiency, severe arrhythmia, etc.; patients concurrent with serious diseases of other systems: chronic lung disease, malignant tumor, severe liver and kidney disease or mental illness; patients can't move or refuse to cooperate; patients admitted blood cell count, blood lipids, liver and kidney function, ions, fasting blood glucose, and high-sensitivity protein measurement routinely.
The anti-human inflammatory factor monoclonal antibody is coated on the enzyme-linked immunosorbent assay (ELISA) plate, and the corresponding inflammatory factor in the human sample will bind to the monoclonal antibody. A biotinylated anti-human inflammatory factor (secondary antibody) antibody is added which will bind to an inflammatory factor bound to the monoclonal antibody to form an immune complex, and the unbound will be washed away. Horseradish peroxidase-labeled Streptavidin will bind to the biotin of the secondary antibody and excess material will be washed away. Color development was carried out by adding 3,3',5,5'-Tetramethylbenzidine (TMB). The concentration of the inflammatory factor is directly proportional to the Optical density $(\mathrm{OD}-450 \mathrm{~nm})$ of the amount of the spectrophotometer, and the plasma concentration of the inflammatory factor of the sample to be tested can be obtained from the standard curve calculated from the standard. Blood samples for the determination of interleukin-6 (IL-6) and interleukin-17A (IL-17A) were collected from the patient's arterial system and the first $3 \mathrm{ml}$ of blood samples were discarded at each sampling. After successful arterial puncture, blood was first collected in the arterial sheath before PCI. In PCI, in order to detect the level of local inflammatory factors in vascular lesions, a 6F Diver CE aspiration catheter was used to locally sample 10-20 mm distal to the coronary stenosis lesion with the support of a guidewire. Finally, blood samples were collected again in the arterial sheath 15 min after stent implantation. Three sampling for each patient takes 45-50 min. All blood samples were slowly withdrawn to minimize activation of the endothelium system to avoid induction of inflammatory response. All blood samples were placed in heparin anticoagulation tubes, and ice soil was immediately placed. Platelet-depleted plasma was obtained by centrifugation at $3000 \mathrm{rpm}$ for $10 \mathrm{~min}$, and frozen in $-71^{\circ}$ refrigerator to be tested. Plasma IL-6, interleukin-9 (IL-9) and IL-17A cytokine concentrations were determined by ELISA (Table 1). Add $100 \mu 1$ Standards, $100 \mu 1$ sample in the corresponding reaction plate hole, gently mix for $30 \mathrm{~s}$, seal the plate hole, incubate at $25^{\circ}$ for $120 \mathrm{~min}$, then wash the plate, drain the liquid in the plate, wash the reaction plate with cleaning solution (300 $\mu$ l of cleaning solution per hole) and remove water droplets (dry on thick stack of absorbent paper) and wash repeatedly 3 times. Then immediately add $100 \mu 1$ of $1 \mathrm{x}$ Biotin to each hole, gently mix for $30 \mathrm{~s}$, seal the plate hole, incubate at $25^{\circ}$ for $60 \mathrm{~min}$ and then wash the plate again, drain the plate, wash the plate with cleaning 
TABLE 1: DETECTION INDICATORS AND TYPES OF MAIN INSTRUMENT KITS

\begin{tabular}{|c|c|}
\hline Detection Indicators & Instrument and Kit Model \\
\hline Flow cytometry & $\begin{array}{c}\text { ACEA NovoCyte Series Flow } \\
\text { Cytometer }\end{array}$ \\
\hline $\begin{array}{l}\text { ELISA enzyme label } \\
\text { analyzer }\end{array}$ & Redux RT-6000 Enzyme Marker \\
\hline IL-9 & $\mathrm{m} 1027278$ \\
\hline IL-17A & m1027486 \\
\hline IL-6 & m1027354 \\
\hline
\end{tabular}

solution ( $300 \mu$ l of cleaning solution per hole). Remove water droplets (dry on thick stack of absorbent paper) and wash 3 times. Repeat the above operation, add $100 \mu 1$ of $1 \mathrm{x}$ horseradish peroxidase (HRP) per hole, mix gently for $30 \mathrm{~s}$, seal the plate hole, and incubate at $25^{\circ}$ for $30 \mathrm{~min}$. Wash the plate: drain the plate and wash the plate with cleaning solution $(300 \mu 1$ of cleaning solution per hole) and remove the water droplets (dry on a thick stack of absorbent paper) and wash it 3 times. Add $100 \mu \mathrm{l}$ of TMB chromogenic solution to each hole again, mix gently for $10 \mathrm{~s}$, and incubate for $15 \mathrm{~min}$ at $25^{\circ}$ in the dark. Finally, add $50 \mu \mathrm{l}$ of Stop Solution to each hole, mix gently for $30 \mathrm{~s}$, read the OD value at $450 \mathrm{~nm}$ in $30 \mathrm{~min}$, draw the standard curve with the OD value as the ordinate and the standard concentration as the abscissa. According to the OD value of the sample, the concentration can be found on the standard curve. The experimental data were processed by Statistical Package for the Social Sciences (SPSS) 21.0 statistical software. The statistical description was performed using the mean \pm standard deviation (mean $\pm \mathrm{SD}$ ) for the parameter test. For the ones that don't meet the parameter test, use the median and the upper and lower quartile 50th percentile (p50) (25th percentile-p25, 75th percentile-p75) for statistical description. For quantitative data that did not obey the normal distribution, two independent sample rank sum tests were used to compare the two. Covariance analysis is used in plasma IL-9 and IL-17A cytokine concentrations in the group of patients undergoing PCI and the control group. The percentage of expression was analyzed by paired $\mathrm{T}$ test in the PCI group flow cytometry. The correlation between the expression of IL-9 and IL-17A and the plasma cytokine concentration in conventional coronary angiography and peripheral blood mononuclear cells did not conform to the normal distribution. Therefore, spearman correlation analysis was used to analyze the correlation between the two. The levels take $a=0.05$ and $a=0.01$. The inflammatory factor levels of the two groups of patients are shown in Table 2. Although the basal levels of plasma IL-6, IL-17A, IL-9 and matrix metalloproteinase-9 (MMP-9) is shown increased in group A, but there was no significant difference compared with group B. No significant differences are shown in the changes of IL6, IL-17A, IL-9 and MMP-9 before and after operation in group B. There was no statistically significant change in the level of MMP-9 in group A compared with the preoperative basal level after PCI, but there was a statistically significant difference in the postoperative increase of IL-6 $(\mathrm{p}=0.025)$. In addition, local IL-6, IL17a and MMP-9 levels were higher than peripheral levels, regardless of before and after surgery $(p<0.001)$. Flow cytometry of patients with acute coronary syndrome within 7-14 d of elective PCI (group A) and conventional coronary angiography (group B) showed the percentage of positive expression of IL-9 cells in peripheral blood mononuclear cells decreased significantly $(p<0.05)$, and the percentage of IL-17A cell positive expression increased significantly $(\mathrm{p}<0.01)$. UL (Double positive) indicates that IL-17A and IL-9 are co-expressed (both positive); LL (Double negative) indicates that IL-17A and IL-9 are not expressed (both negative); UR (Only IL-17A was expressed) indicates IL-17a expression; LR (Only IL-9 was expressed) indicates only IL-9 expression; UL+UR (IL-17A is always expressed) indicates IL-17A expression; UR+IL (IL-9 is always expressed) indicates IL-9 expression (fig. 1). In group B, there was no significant correlation between plasma IL-6 and MMP9 levels in peripheral arteries before and after surgery $(\mathrm{r}=0.15, \mathrm{p}=0.723 ; \mathrm{r}=0.17, \mathrm{p}=0.588)$. However, there was a weak positive correlation between the two indicators in peripheral blood vessels before and after $\mathrm{PCI}$ in PCI group $(\mathrm{r}=0.23, \mathrm{p}=0.053)$. In addition, there was a moderate correlation between the local differences and the preoperative basal levels IL- 6 and MMP-9 of the PCI group $\quad(\mathrm{r}=0.36, \quad \mathrm{p}=0.003)$ (Table 3). It was further found that there was a strong

TABLE 2: CHANGES OF FLOW CYTOMETRY IN PATIENTS WITH PCI AND THOSE WITH ROUTINE CORONARY ANGIOGRAPHY (\%)

\begin{tabular}{lccl}
\hline Flow cytometry & $\begin{array}{c}\text { Group A } \\
(\mathbf{n}=20)\end{array}$ & $\begin{array}{c}\text { Group B } \\
(\mathbf{n}=20)\end{array}$ & $\mathrm{p}$ \\
\hline UL (Double positive) & $0.16 \pm 0.27$ & $0.59 \pm 0.43$ & $<0.001$ \\
UR (Only IL-17A was & $4.35 \pm 5.01$ & $8.97 \pm 6.32$ & 0.01 \\
expressed) & $8.97 \pm 7.70$ & $13.14 \pm 8.26$ & 0.058 \\
$\begin{array}{l}\text { LL (Double negative) } \\
\text { LR (Only IL-9 was }\end{array}$ & $84.28 \pm 7.65$ & $77.38 \pm 7.18$ & 0.001 \\
$\begin{array}{l}\text { expressed) } \\
\text { UR+IL (IL-9 is always }\end{array}$ & $91.23 \pm 9.14$ & $87.25 \pm 9.27$ & 0.036 \\
$\begin{array}{l}\text { expressed) } \\
\text { UL+UR (IL-17A is always } \\
\text { expressed) }\end{array}$ & $4.78 \pm 4.19$ & $8.96 \pm 6.76$ & 0.005 \\
\hline
\end{tabular}


positive correlation between local IL-6 levels and postoperative corrected thrombolysis in myocardial infarction (TIMI) frames in patients with PCI ( $\mathrm{r}=0.61$, $\mathrm{p}<0.001$ ), and stronger correlation between higher than basal levels of local IL-6 activity and postoperative TIMI blood flow frames $(\mathrm{r}=0.63, \mathrm{p}<0.001)$. At the same time, the local MMP-9 higher than the baseline was also correlated with the frames of blood flow after PCI $(\mathrm{r}=0.28, \mathrm{p}=0.038)$ (fig. 2). PCI, as an important means of CHD revascularization therapy, has become one of the effective methods for the treatment of CHD, but complications such as coronary restenosis and myocardial ischemia may occur after interventional therapy, as well as the effect of life quality, psychological disorders and other issues. Inflammatory rehabilitation treatment for CHD after PCI is extremely important from the physiological, psychological, social and other perspectives to improve the quality life of patients. High expression of IL-9 and IL-17A cytokines has been found in many chronic inflammatory diseases, autoimmune diseases, and neoplastic diseases. IL-9 and IL-17A are the major cytokines secreted by T helper-9 (TH9) cells and T helper-17 (TH17) cells. It is found that IL-9 and IL-17A are important cytokines involved in the body's inflammatory response. IL-9 promotes inflammation and allergic reactions by activating macrophages, mast cells and eosinophils, and IL-17A

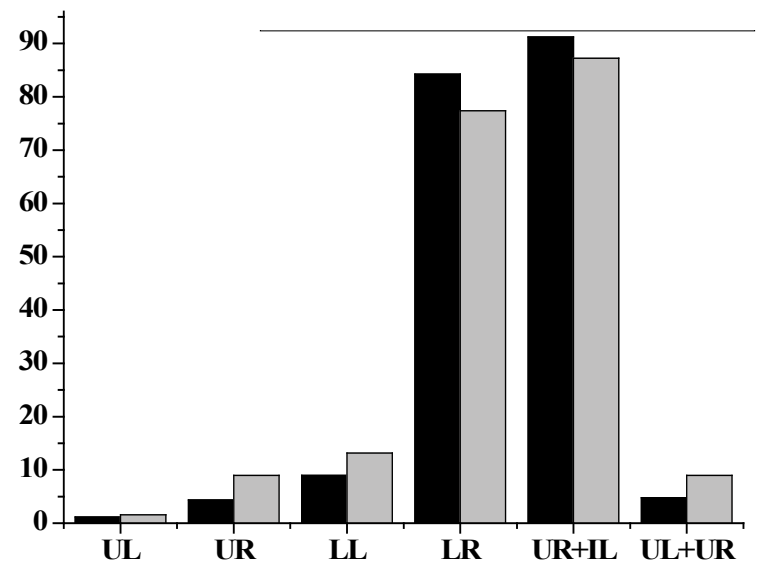

Fig. 1: Changes of flow cytometry in patients undergoing PCI and coronary angiography

TABLE 3: LEVELS OF MMP-9 AND IL-6 IN DIFFERENT PARTS AND TIME

\begin{tabular}{|c|c|c|c|}
\hline & Before surgery & Post-operative & $\mathrm{p}$ \\
\hline \multicolumn{4}{|l|}{ Group A $(n=39)$} \\
\hline MMP-9 (ng/L) & $62.08 \pm 26.34$ & $69.23 \pm 40.02$ & 0.234 \\
\hline IL-6 (pg/ml) & $3.97 \pm 1.93$ & $4.83 \pm 1.24$ & $0.025^{*}$ \\
\hline \multicolumn{4}{|l|}{ Group B $(n=15)$} \\
\hline MMP-9 (ng/L) & $46.5 \pm 30.45$ & $49.23 \pm 41.28$ & 0.496 \\
\hline IL-6 (pg/ml) & $4.56 \pm 1.03$ & $5.12 \pm 0.93$ & 0.211 \\
\hline
\end{tabular}

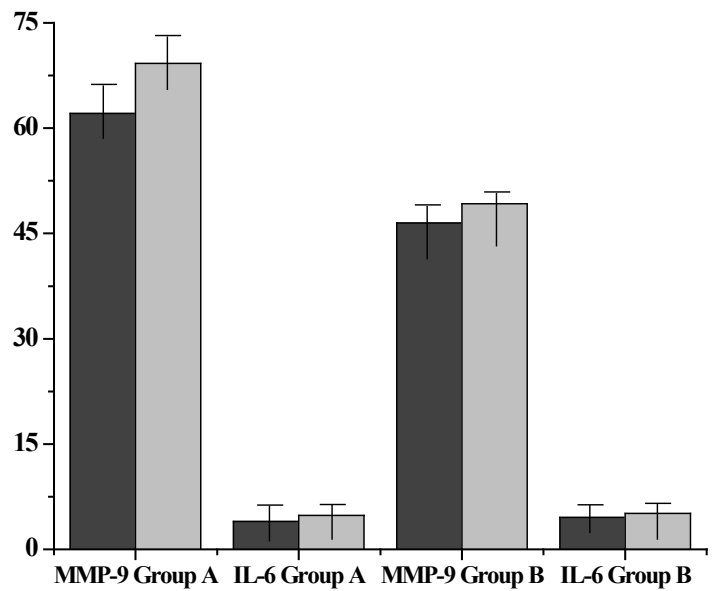

Fig. 2: Comparison of MMP-9 and IL-6 before and after operation

may cause neuro inflammatory responses by inducing activation of astrocytes, microangiopathy, and systemic endotoxin. Therefore, in summary, the study compares the different treatments of PCI and conventional coronary angiography in patients, and finds that the superiority of PCI. Compared with conventional treatments, it can increase the high expression of various cytokines, thereby allowing inflammatory factors such as IL-9 and IL-17A to participate in inflammatory reactions and allergic reactions. Better conditions are provided to reduce complications and improve treatment outcomes. Due to the limitations of the conditions, its one-sidedness and uncertainty is also found during the experiment. Therefore, there is still a long way to go for the diagnosis and rehabilitation of patients with CHD.

\section{Acknowledgement:}

None

\section{Conflict of Interests:}

The authors declared no conflict of interest.

\section{REFERENCES}

1. Sandesara PB, Lambert CT, Gordon NF, Fletcher GF, Franklin BA, Wenger NK, et al. Cardiac rehabilitation and risk reduction: time to "rebrand and reinvigorate". J Am Coll Cardiol 2015;65(4):389-95.

2. Kachur S, Chongthammakun V, Lavie CJ, De Schutter A, Arena R, Milani RV, et al. Impact of cardiac rehabilitation and exercise training programs in coronary heart disease. Prog Cardiovasc Dis 2017;60(1):103-14.

3. Yeh RW, Secemsky EA, Kereiakes DJ, Normand SL, Gershlick $\mathrm{AH}$, Cohen DJ, et al. Development and validation of a prediction rule for benefit and harm of dual antiplatelet therapy beyond 1 year after percutaneous coronary intervention. JAMA 2016;315(16):1735-49.

4. Yang X, Li Y, Ren X, Xiong X, Wu L, Li J, et al. Effects of exercise-based cardiac rehabilitation in patients after 
percutaneous coronary intervention: A meta-analysis of randomized controlled trials. Sci Rep 2017;7:44789.

5. Stergiopoulos K, Boden WE, Hartigan P, Mobius-Winkler $\mathrm{S}$, Hambrecht R, Hueb W, et al. Percutaneous coronary intervention outcomes in patients with stable obstructive coronary artery disease and myocardial ischemia: a collaborative meta-analysis of contemporary randomized clinical trials. JAMA Intern Med 2014;174(2):232-40.

6. Turk-Adawi K, Sarrafzadegan N, Grace SL. Global availability of cardiac rehabilitation. Nat Rev Cardiol 2014;11(10):586.

7. Zarbock A, Schmidt C, Van Aken H, Wempe C, Martens S, Zahn PK, et al. Effect of remote ischemic preconditioning on kidney injury among high-risk patients undergoing cardiac surgery: a randomized clinical trial. Jama 2015;313(21):213341.

8. Shahmansouri N, Farokhnia M, Abbasi SH, Kassaian SE, Tafti AA, Gougol A, et al. A randomized, double-blind, clinical trial comparing the efficacy and safety of Crocus sativus L. with fluoxetine for improving mild to moderate depression in post percutaneous coronary intervention patients. J Affect Disord 2014;155:216-22.

9. Mozaffarian D, Benjamin EJ, Go AS, Arnett DK, Blaha MJ, Cushman M, et al. Executive summary: heart disease and stroke statistics-2016 update: a report from the American Heart Association. Circulation. 2016;133(4):447-54.

This is an open access article distributed under the terms of the Creative Commons Attribution-NonCommercial-ShareAlike 3.0 License, which allows others to remix, tweak, and build upon the work non-commercially, as long as the author is credited and the new creations are licensed under the identical terms

This article was originally published in a special issue, "Trends in Therapeutic Management of Various Clinical Conditions II" Indian J Pharm Sci 2021:83(2)Spl issue;41-45 